\title{
Fetal survival rate after the surgical transfer of two bovine embryos
}

\author{
R. Newcomb*, W. B. Christie† and L. E. A. Rowson \\ A.R.C. Institute of Animal Physiology, Animal Research Station, Cambridge CB3 OJQ, U.K.
}

\begin{abstract}
Summary. Single embryos were bilaterally transferred on Day 7 to the tip of the uterine horn ipsilateral to the corpus luteum and to the tip of the opposite horn, to the tip and contralateral base, base and tip and base and base ( 20 recipients/group). Ten of the recipients in each group were treated with 1000 i.u. hCG daily from Days 13 to 35 after oestrus. At 42 days, pregnancy rate (N.S.) and fetal survival rate $(P<$ 0.05 ) were greater when an embryo had been transferred to the tip rather than to the base of the ipsilateral horn, but the twin pregnancy rate was not improved. Treatment with hCG had no effect on these rates but the corpora lutea of treated animals were heavier $(P<0.02)$.

Unilateral transfers of 2 embryos were made both to the tip of the ipsilateral uterine horn or one to the tip and one to the base (20 recipients/group). The pregnancy rate, fetal survival and twin pregnancy rate in each group were the same, but in the latter one of the embryos had migrated to the other horn in $5 / 9$ animals with twin fetuses. The location of fetuses did not influence their rate of development as determined by fetal measurements.

It is concluded that the tip of the ipsilateral uterine horn is the optimal site for fetal survival and that to ensure a high twin fetal survival one embryo must be in this site.
\end{abstract}

\section{Introduction}

The annual production of calves could be increased without increasing the number of adult female breeding cattle if the rate of twin bearing was greater. A critical requirement which makes the control of twinning by using gonadotrophins more exacting is that one egg must be shed from each ovary if a high twin pregnancy rate is to be achieved (Gordon, Williams \& Edwards, 1962). Confirmation of this observation has come from surgical embryo transfer studies made by Rowson, Lawson \& Moor (1971), who showed that a high twin-pregnancy rate was achieved (73\% of pregnant recipients) after bilateral transfer of a single embryo to each uterine horn, but not after the unilateral transfer of two embryos to the uterine horn ipsilateral to the corpus luteum (45\%). This appeared to be the result of competition between embryos within a single uterine horn. Other workers have confirmed the high pregnancy and twinning rate which may be achieved after bilateral embryo transfer (Sreenan, Beehan \& Mulvehill, 1975) which appears therefore to be the method of choice for inducing twin pregnancy. In practice, because economic considerations preclude the use of surgery, twinning would only be induced by non-surgical

- Present address: Milk Marketing Board (England \& Wales), Thames Ditton, Surrey, U.K.

$\dagger$ Present address: CBS (Embryos) Ltd, Vallum Farm, Military Road, Stamfordham, Newcastle-upon-Tyne NE18 OLL, U.K. 
methods: (1) one embryo may be placed non-surgically into the contralateral horn of a previously inseminated recipient (Boland, Crosby \& Gordon, 1975; Boland \& Gordon, 1978; Sreenan, 1978) or (2) one embryo may be transferred into each uterine horn (Heyman, Renard, Ozil \& du Mesnil du Buisson, 1978). Relative to surgery neither method has been very successful; the pregnancy rate with both methods has been similar to that after artificial insemination alone (approximately 50\%) but fetal survival of the transferred embryos has been quite low (25-43\%).

In contrast, when single embryos have been transferred non-surgically to the ipsilateral horn of unmated recipients the fetal survival rate has been greater (approximately 50\%) (Trounson, Rowson \& Willadsen, 1978; Jillella \& Baker, 1978; Newcomb, 1979; Christie, Newcomb \& Rowson, 1980). However, when an embryo is transferred non-surgically to the uterine horn contralateral to a surgical transfer (which has an $85 \%$ fetal survival), its survival is low (35\%) (Newcomb, Christie \& Rowson, 1978b). If a high pregnancy and twinning rate can be achieved by the surgical transfer of an embryo to the tip of each uterine horn, it is not obvious why an embryo transferred non-surgically to the horn contralateral to a native or a surgically transferred embryo does not result in a similarly high twinning rate. Because non-surgically transferred embryos are usually deposited in the cervical third (base) of the uterine horn while surgical transfers are to the ovarian tip of the horn, there could be a location effect on the transferred embryo or simply some adverse effect of the non-surgical method itself.

The survival of a single embryo transferred to the contralateral horn of an unmated recipient is low (Newcomb \& Rowson, 1976; Sreenan, 1976; Christie, Newcomb \& Rowson, 1979) and survival in the contralateral horn of a bred recipient is largely dependent on the viability of the native egg. However, treatment with hCG increases pregnancy rate after surgical transfer only to the contralateral uterine horn (Christie et al., 1979).

In this study we have surgically transferred an embryo to each uterine horn and examined the effect of site of transfer. Half of the recipients were treated with hCG in the belief that this would increase pregnancy rate and thereby demonstrate more positively competition between embryos which was a consequence of their location. We also examined the result of transfer of two embryos to the ipsilateral uterine horn.

\section{Materials and Methods}

Experimental animals were heifers of mixed origin but were predominantly Hereford/Friesian crossbreeds. Embryos were recovered non-surgically in phosphate-buffered saline (PBS: Whittingham, 1971) at Day 7 after oestrus using the method described by Newcomb, Christie \& Rowson (1978a). Recipient heifers were in oestrus synchronously or \pm 1 day asynchronously with the embryo donors. They were fasted for $24 \mathrm{~h}$, anaesthetized, and placed in ventral recumbency. The uterus was exposed through a mid-ventral incision (Rowson, Moor \& Lawson, 1969).

Embryos were transferred to the uterus in $0.025-0.05 \mathrm{ml}$ PBS using a fine Pasteur pipette. The pipette was introduced through a small puncture made into the lumen of the uterus with a blunt 18-gauge needle. For transfers to the tip of the uterus the puncture was made $2.5-3.5 \mathrm{~cm}$ from the utero-tubal junction and the pipette was directed towards the cervix before expelling the embryo. For transfers to the base, the uterus was punctured in the region of the anterior extremity of the intercornual ligament and the pipette was directed towards the cervix.

Recipients were slaughtered 35 days after embryo transfer (i.e. 42 days after donor oestrus). The surviving fetuses were exposed and the location, length of the amniotic sac and crown-rump length of each were noted. The normality of the fetus was assessed using the criteria of Christie et al. (1979) and the fresh weight of the corpus luteum of pregnant animals was recorded. Degenerating embryos were noted but not included in the analysis of results. 


\section{Experiment 1}

One embryo was transferred to each uterine horn of 80 recipients. The recipients were equally allocated to 4 groups and embryos were transferred where possible as replicates to the tip of the uterine horn ipsilateral to the corpus luteum and the tip of the contralateral horn $(\mathrm{t} / \mathrm{t})$, the tip ipsilaterally and the base contralaterally $(t / b)$, the base ipsilaterally and the tip contralaterally $(b / t)$, or the base ipsilaterally and contralaterally $(b / b)$. In each group 10 recipients were treated with 1000 i.u. hCG from Days 13 to 35 (Christie et al., 1979).

\section{Experiment 2}

Two groups of 20 recipients received either 2 embryos to the tip of the ipsilateral uterine horn $(2 \mathrm{t})$ or one embryo to the tip and one at the base of the ipsilateral horn $(1 \mathrm{t}, 1 \mathrm{~b})$.

\section{Results}

\section{Experiment 1}

As shown in Table 1, the highest pregnancy rates occurred when the ipsilateral transfer was made to the tip of the uterine horn $(t / t=90 \% ; t / b=85 \% ; b / t=75 \% ; b / b=65 \%)$. The highest overall fetal survival was also achieved when an embryo had been placed at the ipsilateral tip $(58 / 80)$ compared with ipsilateral base transfer $(46 / 80)\left(\chi^{2}\right.$ with continuity correction $=3 \cdot 32$; d.f. $=1 ; P=<0.05$, one-tailed test). Survival in the contralateral horn was unaffected by location in that horn but was greatest when an embryo had been transferred to the ipsilateral tip $(27 / 40)$ and least when the ipsilateral transfer was made to the base of the horn $(22 / 40)$. The fetal survival at each transfer site was 31 at the ipsilateral tip, 24 at the ipsilateral base, 24 at the contralateral tip and 25 at the contralateral base. There was no effect of hCG treatment on fetal survival rate compared with untreated controls ( $51 / 80$ and 53/80 respectively). The highest twin pregnancy rate occurred when transfer had been made to the tip of the ipsilateral horn $(t / t$ and $t / b$ ) but when expressed as a percentage of animals pregnant groups were comparable (Table 1).

Table 1. Fetal survival at Day 42 in heifers (20/group) after transfer on Day 7 of a single embryo to the tip ( $t$ ) or base (b) of the uterine horn ipsilateral or contralateral to the corpus luteum

\begin{tabular}{lllll}
\hline & Group $\mathrm{t} / \mathrm{t}$ & Group $\mathrm{t} / \mathrm{b}$ & Group b/t & Group b/b \\
\hline $\begin{array}{l}\text { No. of pregnant recipients } \\
\begin{array}{l}\text { No. of fetuses } \\
\text { Ipsilateral horn }\end{array}\end{array}$ & 18 & 17 & 15 & 13 \\
$\quad$ Contralateral horn & $16+1 \mathrm{~d}$ & $15+1 \mathrm{~d}$ & 12 & 12 \\
$\quad$ Total (\% survival) & 13 & 14 & $11+1 \mathrm{~d}$ & 11 \\
$\begin{array}{l}\text { No. of recipients with live } \\
\quad \text { twin fetuses }\end{array}$ & $29(72.5)$ & $29(72.5)$ & $23(57.5)$ & $23(57.5)$ \\
As \% of recipients & 11 & 12 & & 10 \\
As \% of pregnant recipients & 55 & 60 & 40 & 50 \\
\hline
\end{tabular}

$\mathrm{d}=$ degenerate fetus.

- One embryo had migrated from the other horn or was erroneously transferred ipsilaterally.

\section{Experiment 2}

There was no difference in pregnancy rate, fetal survival rate or twin pregnancy rate between groups (Table 2). In Group 1t,1b, one fetus had migrated to the contralateral uterine horn in a high proportion of the animals pregnant with twin fetuses (5/9). 
Table 2. Fetal survival at Day 42 in heifers (20/group) after transfer on Day 7 of 2 embryos to the tip (2t) or one to the tip and one to the base

$(1 t, 1 b)$ of the uterine horn ipsilateral to the corpus luteum

\begin{tabular}{lcc}
\hline & Group 2t & Group 1t, 1b \\
\hline $\begin{array}{l}\text { No. of pregnant recipients } \\
\text { No. of fetuses }\end{array}$ & 16 & 15 \\
$\quad$ Ipsilateral horn & $24+4 \mathrm{~d}$ & $19+2 \mathrm{~d}$ \\
$\quad$ Contralateral horn (\%) & $1(4)$ & $5+1 \mathrm{~d}(25)$ \\
$\quad$ Total (\%) & $25(62.5)$ & $24(60 \cdot 0)$ \\
No. of recipients & 9 & \\
$\quad$ With viable twin fetuses & $1(11 \cdot 1)$ & 5 \\
$\quad$ With 1 twin in contralateral horn (\%) & 45 & 45 \\
As \% of recipients & 56.3 & 56.3 \\
As \% of pregnant recipients & & \\
\hline
\end{tabular}

$\mathrm{d}=$ degenerate fetus.

In both experiments there was no difference in the length of the amniotic sac or crown-rump length of the fetus that could be related to the site of embryo transfer or the presence of single or twin fetuses (e.g. crown-rump length, ipsilateral tip $2.7 \pm 0.04 \mathrm{~cm}$ compared with other sites, $2.7 \pm 0.03 \mathrm{~cm}$; twin fetuses $2.70 \pm 0.02 \mathrm{~cm}$ and single fetuses $2.76 \pm 0.04 \mathrm{~cm}$ )

There was no difference between the weight of the corpus luteum when located in the left $(6.4 \pm 0.3 \mathrm{~g})$ or in the right $(6.0 \pm 0.2 \mathrm{~g})$ ovary of pregnant animals or from animals with single or single + a degenerate fetus $(6 \cdot 1 \pm 0.4 \mathrm{~g})$ and those carrying two fetuses $(6.2 \pm 0.2 \mathrm{~g})$. There was no effect on corpus luteum weight after the transfer of an embryo to the ipsilateral tip compared with the ipsilateral base or when a fetus was present in the ipsilateral horn at slaughter when compared with pregnant animals without an ipsilateral fetus. However, the corpora lutea of pregnant animals which had been treated with hCG from Days 13 to 35 were significantly ( $P$ $<0.02)$ heavier $(7.2 \pm 0.3 \mathrm{~g})$ than those of untreated controls $(6.0 \pm 0.3 \mathrm{~g})$.

\section{Discussion}

The tendency for higher overall fetal survival to occur after transfer to the tip of the ipsilateral uterine horn rather than its base supports other studies which have indicated a site effect within the ipsilateral horn after single embryo transfer (Newcomb, Christie \& Rowson, 1978c; Christie et al., 1980), although a bilateral transfer study either to the tip or base of the uterus did not previously demonstrate any differences (Sreenan, 1976). It is most unlikely that any technical difficulty in transferring to the base of the uterine horn has influenced the results of this study since the fetal survival rate at each site other than the ipsilateral tip was identical.

The original premise of the study was based on findings which indicated that non-surgical transfer to the contralateral horn resulted in a low fetal survival rate in that horn despite a high survival of surgically transferred embryos in the ipsilateral horn (Newcomb et al., 1978b). It has also been shown that a high proportion of embryos transferred non-surgically to the ipsilateral horn survive at least until the normal time of luteolysis (Christie et al., 1980). Consequently, the poor performance of embryos transferred non-surgically with those transferred surgically was best explained either by their location in the base of the contralateral horn or a competitive effect between the two embryos related to their situation or method of transfer.

The present study shows that site itself does not seem to have an effect on fetal survival except within the ipsilateral horn. Competition between the two embryos after bilateral transfer resulting from their location alone seems most unlikely. It seems more probable that some effect of the non-surgical transfer method might itself retard development sufficiently for competition to influence survival of the non-surgically transferred embryo. 
Unlike previous findings (Christie et al., 1979, 1980) there was no increase in the proportion of fetuses surviving after treating recipients with hCG. It may be that the luteotrophic/antiluteolytic effect of two embryos is itself adequate to replace the beneficial function of hCG on fetal survival which has been demonstrated after single embryo transfer to an unsuitable site, but that, despite hCG treatment, the location of the embryo has a specific effect on its survival. Although the administration of hCG affected the weight of the corpus luteum there was no differential effect of location of the fetus or two fetuses compared with one fetus on luteal weight. This suggests that the fetus may not be acting luteotrophically at this stage but antiluteolytically. The consistency of the fetal measurements in different sites, and in single or twin pregnancies agrees with previous observations (Sreenan \& Beehan, 1976) which showed little variation up to 50 days after oestrus.

In Exp. 2 both groups had similar pregnancy, fetal survival and twin pregnancy rates to one another and to Groups $b / t$ and $b / b$ in Exp. 1. The rate of twin pregnancy was higher than after unilateral transfer in the study of Rowson et al. (1971) in which pregnancy was diagnosed by slaughter at 60 or 90 days after oestrus or at calving. In this study pregnancy was diagnosed by slaughter at Day 42, but it is unlikely that much loss would have occurred after this time (Sreenan \& Beehan, 1976).

A high proportion of the embryos in Group lt, lb in Exp. 2 migrated (55\% of those pregnant with twins). In consequence, and because of the similar results for Groups $b / t$ and $b / b$ in Exp. 1 , it would seem logical to transfer non-surgically to the ipsilateral horn of a bred recipient. Compared with transfer to the contralateral horn this might lead to an increase in the pregnancy rate without affecting the number of twins, unless non-surgical transfer renders an embryo less able to compete with the native egg. Whether this will be the best method for obtaining twin pregnancies non-surgically or whether one embryo is best transferred non-surgically to each horn can only be ascertained by further study of non-surgical transfers. In any event nonsurgical transfer is unlikely to produce as high a pregnancy rate as the surgical transfer of embryos to each uterine tip unless the embryos can be delivered to these sites without trauma.

R.N. is seconded from the Milk Marketing Board (England and Wales).

\section{References}

Boland, M.P. \& Gordon, I. (1978) Twinning in lactating Friesian cows by non-surgical egg transfer, Vet. Rec. 103, 241.

Boland, M.P., Crosby, T.F. \& Gordon, I. (1975) Twin pregnancy in cattle established by non-surgical egg transfer. Brit. vet. J. 131, 738-740.

Christie, W.B., Newcomb, R. \& Rowson, L.E.A. (1979) Embryo survival in heifers after transfer of an egg to the uterine horn contralateral to the corpus luteum and the effect of treatments with progesterone or hCG on pregnancy rates. $J$. Reprod. Fert. 56, 701-706.

Christie, W.B., Newcomb, R. \& Rowson, L.E.A. (1980) Non-surgical transfer of bovine eggs: Investigation of some factors affecting embryo survival. Vet. Rec. (in press).

Gordon, I., Williams, G. \& Edwards, J. (1962) The use of serum gonadotrophin (PMS) in the production of twin pregnancy in the cow. J. agric. Sci., Camb. 59, $143-198$.

Heyman, Y., Renard, J.-P., Ozil, J.-P. \& du Mesnil du Buisson, F. (1978) Cervical embryo transfer at different stages in cattle. In Control of Reproduction in the Cow, pp. 330-335. Ed. J. M. Sreenan.
Commission of the European Communities, Luxembourg.

Jillella, D. \& Baker, A.A. (1978) Transcervical transfer of bovine embryos. Vet. Rec. 103, 574-576.

Newcomb, R. (1979) Surgical and non-surgical transfer of bovine embryos. Vet. Rec. 105, 432-434.

Newcomb, R. \& Rowson, L.E.A. (1976) Aspects of the non-surgical transfer of bovine eggs. Proc. 8th Int. Congr. Anim. Reprod. \& A.I., Krakow, vol. 3, pp. 262-265.

Newcomb, R., Christie, W.B. \& Rowson, L.E.A. (1978a) Non-surgical recovery of bovine embryos. Vet. Rec. $102,414-417$.

Newcomb, R., Christie, W.B. \& Rowson, L.E.A. (1978b) Comparison of the fetal survival rate in heifers after the transfer of an embryo surgically to one uterine horn and non-surgically to the other. J. Reprod. Fert. 52, 395-397.

Newcomb, R., Christie, W.B. \& Rowson, L.E.A. (1978c) The non-surgical recovery and transfer of bovine embryos. In Control of Reproduction in the Cow, pp. 292-304. Ed. J. M. Sreenan. Commission of the European Communities, Luxembourg. 
Rowson, L.E.A., Moor, R.M. Lawson, R.A.S. (1969) Fertility following egg transfer in the cow; effect of method, medium and synchronisation of oestrus. $J$. Reprod. Fert. 18, 517-523.

Rowson, L.E.A. Lawson, R.A.S. \& Moor, R.M. (1971) Production of twins in cattle by egg transfer. $J$. Reprod. Fert. 25, 261-268.

Sreenan, J.M. (1976) Egg transfer in the cow: Effect of site of transfer. Proc. 8th Int. Congr. Anim. Reprod. \& A.I., Krakow, vol. 3, pp. 269-272.

Sreenan, J.M. (1978) Non-surgical egg recovery and transfer in the cow. Vet. Rec. 102, 58-60.

Sreenan, J.M. \& Beehan, D. (1976) Embryonic survival and development at various stages of gestation after bilateral egg transfer in the cow. J. Reprod. Fert. 47, 127-128.

Sreenan, J.M., Beehan, D. \& Mulvehil, P. (1975) Egg transfer in the cow: Factors affecting pregnancy and twinning rates following bilateral transfers. $J$. Reprod. Fert. 44, 77-85.

Trounson, A.O., Rowson, L.E.A. W Wlladsen, S.M. (1978) Non-surgical transfer of bovine embryos. Vet. Rec. 102, 74-75.

Whittingham, D.G. (1971) Survival of mouse embryos after freezing and thawing. Nature, Lond. 233, 125126.

Received 13 August 1979 